\title{
Two coordination polymers containing the dicyanamide ligand: synthesis, crystal structures, and HFEPR studies
}

\author{
Lívia B. L. Escobar ${ }^{a, b}$, Charlane C. Corrêa ${ }^{a}$, Guilherme P. Guedes ${ }^{c}$, Maria G.F. Vaz \\ Samuel M. Greer ${ }^{\mathrm{d}, \mathrm{e}}$, Stephen Hill, ${ }^{\mathrm{d}, \mathrm{f}}$ Renata Diniz ${ }^{\mathrm{a}}$, Flávia C. Machado ${ }^{\mathrm{a}, *}$
}

\begin{abstract}
a'Departamento de Química, Universidade Federal de Juiz de Fora, Campus Universitário s/n, Martelos, Juiz de Fora, MG 36036-900, Brazil

'Instituto de Química, Universidade Federal Fluminense, Niterói - RJ, 24020-150, Brazil

' Instituto de Ciências Exatas, Departamento de Química, Universidade Federal Rural do Rio de Janeiro, Seropédica - RJ, 23.890-000, Brazil.

${ }^{d}$ National High Magnetic Field Laboratory, Florida State University, Tallahassee, FL 32310, United States.

eDepartment of Chemistry, Florida State University, Tallahassee, FL 32306, United States.

fDepartment of Physics, Florida State University, Tallahassee, FL 32306, United States.

* Corresponding author. Tel.: +55 32 21023310; fax: +55 3221023314.

E-mail address: flavia.machado@ufjf.edu.br (F.C. Machado).
\end{abstract}

\section{Abstract}

Two new compounds [Ni(dca $\left.)_{2}(\mathrm{dmdpy})\right]$ (1) and [Zn(dca $\left.)_{2}(\mathrm{dmdpy})\right](\mathbf{2}),(\mathrm{dca}=$ dicyanamide and dmdpy = 5,5'-dimethyl-2,2'-dipyridine) were synthesized and characterized by single crystal X-ray diffraction, FTIR and thermal analysis. In both compounds, the dmdpy ligand adopts a bidentate chelating coordination mode while dca anions act in a $\mu_{1,5}$ bridging fashion. $\ln \mathbf{1}$, the $\mathrm{Ni}^{\prime \prime}$ centers lie on a distorted octahedral geometry, whereas in $\mathbf{2}$ the $\mathrm{Zn}$ " geometry is distorted square pyramidal. In compounds 1 and $\mathbf{2}$, dca ligands connect the metal sites giving rise to 2D and 1D coordination polymers, respectively. The magnetic properties of 1 were studied by magnetic susceptibility and high field/frequency electron paramagnetic resonance (HFEPR) measurements.

Keywords: Coordination polymers, crystal structure, magnetic properties.

\section{Introduction}

Recently, the construction of supramolecular systems of metal-organic frameworks and coordination polymers has been a subject of significant interest.[1] The realm of crystal engineering has consequently achieved great success in the assembly of such systems. In addition to the resultant crystalline materials having diverse structures these materials display useful properties in gas storage, catalysis, ion exchange, optical, electric and molecular magnetism.[2-4]

The design, synthesis, and structural characterization of coordination polymers have been intensely investigated over the past two decades.[4, 5] Common spacers used for the connection of transition metals to polymeric structures include cyanides, azides, cyanates, thiocyanates, selenocyanates, dicyanamides, tricyanomethanides, N-heterocyclic auxiliary 
ligands together with related anionic ligands such as tetracyanoethanide or tetracyanoquinodimethanide and many others.[6, 7]

It was proved that pseudohalides could behave as bridging ligands connecting transition metals to give complexes with various topological structures, including isolated molecular clusters, 1D chains, and 2D or 3D networks.[8, 9] Another advantage of using this ligand's family as spacers is their ability to effectively transmit magnetic coupling between the spin carriers through the delocalized $p$-bond skeleton of the bridging unit.[6]

In particular, the cyano group has been used extensively as a versatile mediator for magnetic coupling in molecule-based magnets.[10] The larger pseudohalide ligand dicyanamide (dca) has three potential nitrogen-donor sites and coordinates to metal ions in various modes (see Scheme 1). These different coordination modes allow for the preparation of compounds with various molecular architectures including mono- and polynuclear systems, as well as one-, two- and three-dimensional networks.[5, 11, 12]

Herein, we report the syntheses and crystal structures of two coordination polymers, $\left[\mathrm{Ni}(\mathrm{dca})_{2}(\mathrm{dmdpy})\right]_{\mathrm{n}}(\mathbf{1})$, and $\left[\mathrm{Zn}(\mathrm{dca})_{2}(\mathrm{dmdpy})\right]_{\mathrm{n}}(\mathbf{2})$. Compound $\mathbf{1}$ is isostructural to the $\left[\mathrm{Co}(\mathrm{dca})_{2}(\mathrm{dmdpy})\right]_{\mathrm{n}}$ described by us previously.[13] In the present work, we used HFEPR spectroscopy to characterize the magnetic anisotropy of 1.
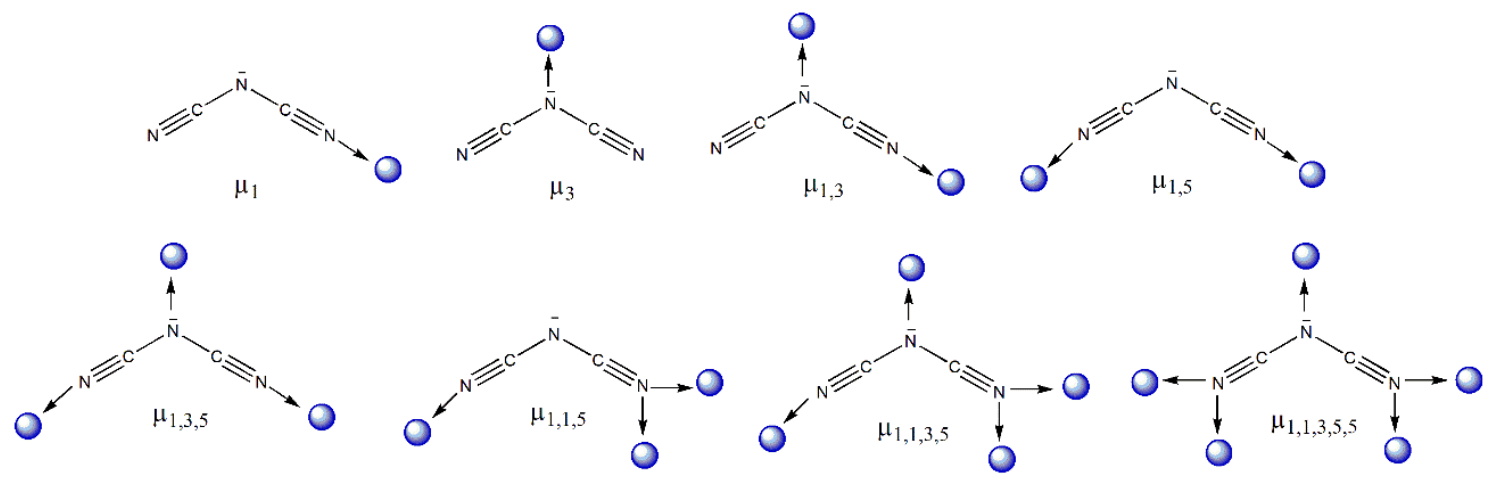

O Metal ion

Scheme 1- Coordination modes of the dca ligand.

\section{Experimental}

\subsection{Materials and methods}

Analytical grade solvents and reagents were purchased from commercial sources and used without further purification. The elemental analyses ( $\mathrm{CHN})$ were carried out on a PerkinElmer 2400 Series II analyzer. Infrared spectra were recorded on a FT-IR Bomen MB102 spectrometer in the frequency range $4000-400 \mathrm{~cm}^{-1}$ with an average of $128 \mathrm{scans}$ and $4 \mathrm{~cm}^{-1}$ of spectral resolution using $\mathrm{KBr}$ pellets. Thermal analysis (TG/DTA) for $\mathbf{1}$ and $\mathbf{2}$ were performed 
using a TA instruments SDT Q600 using nitrogen gas purge atmosphere at $50 \mathrm{~mL} \mathrm{~min}^{-1}$ and a heating rate of $10^{\circ} \mathrm{C} \mathrm{min}^{-1}$ in a range from 25 to $1000{ }^{\circ} \mathrm{C}$.

Single crystal X-ray data were collected using an Oxford GEMINI A Ultra diffractometer with Mo Ka $(\lambda=0.71073 \AA)$ radiation at room temperature $(298 \mathrm{~K})$. Data collection, reduction, and cell refinement were performed using the CrysAlis RED program, Oxford diffraction Ltda Version 1.171.32.38.[14] The structures were solved and refined using SHELXS-97 and SHELXL-97 packages.[15] An empirical isotropic extinction parameter $x$ was refined according to the method described by Larson;[16] a multiscan absorption correction was applied.[17] The structures were drawn by VESTA[18] and Mercury[19] programs. Details of data collection and structure refinement for compounds $\mathbf{1}$ and $\mathbf{2}$ are summarized in Table 1.

Magnetic measurements were performed on a Cryogenic SX-600 SQUID magnetometer in the range from 2-290 $\mathrm{K}$ with an applied field of $0.1 \mathrm{~T}$. The samples were placed in a gelatin capsule and data were corrected for the diamagnetism and sample holder.

High-field, high-frequency EPR measurements were carried out at the National High Magnetic Field Laboratory(NHMFL), in Tallahassee, Florida. Spectra were collected on a constrained powder of 1 over a temperature range of ca. $5 \mathrm{~K}$ to $30 \mathrm{~K}$. The spectrometer is a transmission-type device in which microwaves are propagated through cylindrical light pipes.[20] The microwaves were generated by a phase-locked Virginia Diodes source along with a series of frequency multipliers. A superconducting magnet (Oxford Instruments) capable of reaching a field of $17 \mathrm{~T}$ was employed.

\subsection{Synthesis}

$\left[\mathrm{Ni}(\mathrm{dca})_{2}(\mathrm{dmdpy})\right]_{\mathrm{n}}$ (1). A methanolic solution $(5 \mathrm{~mL})$ of $\mathrm{NaN}(\mathrm{CN})_{2}(0.79 \mathrm{mmol})$ was added dropwise to an aqueous solution of $\mathrm{Ni}\left(\mathrm{NO}_{3}\right)_{2} \cdot 6 \mathrm{H}_{2} \mathrm{O}(0.39 \mathrm{mmol})$. Dmdpy $(0.27 \mathrm{mmol})$ dissolved in $\mathrm{MeOH}(5 \mathrm{~mL})$ was slowly added to the reaction mixture. Two days later blue single crystals were obtained and collected by filtration. Yield: $38 \%$. Anal. Calc. for $\mathrm{C}_{16} \mathrm{H}_{12} \mathrm{~N}_{8} \mathrm{Ni}$ : C, 51.2; $\mathrm{H}$, 3.2; N, $29.8 \%$. Found: C, 50.3; H, 3.2; N, $28.6 \%$. IR $\left(\mathrm{KBr}, \mathrm{cm}^{-1}\right): v_{\mathrm{as}}+v_{\mathrm{s}}(\mathrm{C} \equiv \mathrm{N}) 2310 ; v_{\mathrm{as}}(\mathrm{C} \equiv \mathrm{N})$ 2255; $v_{s}(C \equiv N)$ 2219, 2187; $v_{C C / C N} 1608,1574,1504,1479 ; v_{a s}(C-N) 1365$ and $v_{s}(C-N) 927$.

[Zn(dca) $\left.{ }_{2}(\mathrm{dmdpy})\right]_{n}(\mathbf{2})$. This compound was synthesized as described for compound 1 except for the use of $\mathrm{ZnCl}_{2}(0.39 \mathrm{mmol})$. Two days later colorless crystals were obtained and collected by filtration Yield 35\%. Anal. Calc. for $\mathrm{C}_{16} \mathrm{H}_{12} \mathrm{~N}_{8} \mathrm{Zn}$ : C, 50.3; H, 3.2; N, 29.4\%. Found: C, 50.2; H, 3.1; N, 29.2\%. IR $\left(\mathrm{KBr}, \mathrm{cm}^{-1}\right): v_{\mathrm{as}}+v_{\mathrm{s}}(\mathrm{C} \equiv \mathrm{N}) 2338 ; v_{\mathrm{as}}(\mathrm{C} \equiv \mathrm{N}) 2258 ; v_{\mathrm{s}}(\mathrm{C} \equiv \mathrm{N}) 2227$, 2182; $v_{\mathrm{CC} / \mathrm{CN}} 1609,1581,1484 ; v_{\mathrm{as}}(\mathrm{C}-\mathrm{N}) 1347$; and $v_{\mathrm{s}}(\mathrm{C}-\mathrm{N}) 929$. 


\section{Results and discussion}

\subsection{General Characterization}

IR spectra (Fig. S1 in the supplementary material) exhibit dca stretching vibrations for 1 and 2 as strong absorptions in the region 2182-2227 $\mathrm{cm}^{-1}$ and two medium to strong absorptions in the range of $2255-2260 \mathrm{~cm}^{-1}$ and $2283-2310 \mathrm{~cm}^{-1}$, which are attributed to $v_{s}(C \equiv N), v_{a s}(C \equiv N)$ and $v_{a s}+v_{s}(C \equiv N)$ modes, respectively. The shifts towards higher frequency values, when compared to the free dca ligand $\left(2170,2232\right.$ and $\left.2286 \mathrm{~cm}^{-1}\right)$ are presumably due to the bidentate bridging $\left(\mu_{1,5}\right)$ coordination mode of the pseudohalide.[21-23] Bands corresponding to $v_{a s}(C-N)$ and $v_{s}(C-N)$ stretching frequencies are found in the ranges 13651392 and $927-929 \mathrm{~cm}^{-1}$, respectively. The presence of bands in the region $1608-1479 \mathrm{~cm}^{-1}$ is assigned to $v(\mathrm{CC} / \mathrm{CN})$ of dmdpy ligand pyridyl rings. These bands were shifted to larger frequencies when compared to the free ligand $\left(1590-1490 \mathrm{~cm}^{-1}\right)$ indicating the pyridyl coordination toward the metal centers.

In order to examine thermal stabilities of $\mathbf{1}$ and $\mathbf{2}$, thermogravimetric and differential thermal analyses (TG/DTA) were carried out in dynamic nitrogen atmosphere. TG/DTA curves (Fig. S2 in the supplementary material) indicate that compounds $\mathbf{1}$ and 2 are stable up to 329 and 216 ${ }^{\circ} \mathrm{C}$, respectively. Above these temperatures thermal decomposition of the complexes occurs gradually in several steps through endothermic and exothermic events. In the case of compound 1, the percentage residue (Exp., $23.1 \%$, Calc., 20,4 \%) agrees with metallic nickel and carbonized material. The percentage residue $(28.2 \%)$ at $900{ }^{\circ} \mathrm{C}$ for compound 2 indicates an incomplete decomposition at this temperature. 
Table 1- Crystallographic data for $\left[\mathrm{Ni}(\mathrm{dca})_{2}(\mathrm{dmdpy})\right]_{\mathrm{n}}(\mathbf{1})$ and $\left[\mathrm{Zn}(\mathrm{dca})_{2}(\mathrm{dmdpy})\right]_{\mathrm{n}}(\mathbf{2})$.

\begin{tabular}{|c|c|c|}
\hline Compound & {$\left[\mathrm{Ni}(\mathrm{dca})_{2}(\mathrm{dmdpy})\right]_{\mathrm{n}}$} & {$\left[\mathrm{Zn}(\mathrm{dca})_{2}(\mathrm{dmdpy})\right]_{\mathrm{n}}$} \\
\hline Formula & $\mathrm{C}_{16} \mathrm{H}_{12} \mathrm{NiN}_{8}$ & $\mathrm{C}_{16} \mathrm{H}_{12} \mathrm{Zn} \mathrm{N} \mathrm{N}_{8}$ \\
\hline Formula weight $\left(\mathrm{g} \mathrm{mol}^{-1}\right)$ & 375.05 & 381.71 \\
\hline Crystal system & Orthorhombic & Triclinic \\
\hline Space group & Pccn & $P-1$ \\
\hline \multicolumn{3}{|l|}{ Unit cell dimensions } \\
\hline$a(\AA)$ & $8.1716(2)$ & $7.5469(3)$ \\
\hline$b(\AA)$ & $12.3047(2)$ & $9.3700(3)$ \\
\hline$c(\AA)$ & $17.6924(4)$ & $12.8380(4)$ \\
\hline$\alpha\left(^{0^{\prime}}\right)$ & 90.00 & $101.219(3)$ \\
\hline$\beta\left(^{\circ}\right)$ & 90.00 & $102.340(3)$ \\
\hline$\gamma\left(0^{\circ}\right)$ & 90.00 & $104.515(3)$ \\
\hline$V\left(\AA^{3}\right)$ & $1778.95(7)$ & $828.47(5)$ \\
\hline z & 4 & 2 \\
\hline Crystal size $(\mathrm{mm})$ & $0.605 \times 0.260 \times 0.154$ & $0.315 \times 0.259 \times 0.066$ \\
\hline dcalc $\left(\mathrm{g} \mathrm{cm}^{-3}\right)$ & 1.400 & 1.530 \\
\hline$\mu(\operatorname{Mo~K\alpha })\left(\mathrm{cm}^{-1}\right)$ & 1.106 & 1.499 \\
\hline $\mathrm{T}_{\min } / \mathrm{T}_{\max }$ & $0.872 / 1.000$ & $0.954 / 1.000$ \\
\hline Measured reflections & 76216 & 18030 \\
\hline Unique reflections & 1825 & 3384 \\
\hline Observed reflections ${ }^{a}$ & 1416 & 2617 \\
\hline No. parameters & 115 & 226 \\
\hline$R^{b}$ & 0.0367 & 0.0452 \\
\hline$w R^{a}$ & 0.0949 & 0.0985 \\
\hline $\mathrm{s}$ & 1.107 & 1.067 \\
\hline RMS peak $\left(\mathrm{e} \AA^{-3}\right)$ & 0.061 & 0.072 \\
\hline
\end{tabular}

${ }^{\mathrm{a}}\left[F_{o}^{2}>2 \sigma F_{o}^{2}\right]$

${ }^{\mathrm{b}}\left[\mathrm{F}_{\mathrm{o}}>2 \sigma \mathrm{F}_{\mathrm{o}}\right]$

\subsection{Structure descriptions}

It was possible to observe that the use of the $\mathrm{Ni}^{\text {II }}$ ion favors the formation of a $2 \mathrm{D}$ coordination polymer, whilst the use of $\mathrm{Zn}^{\prime \prime}$ contributes to obtaining a $1 \mathrm{D}$ coordination polymer (scheme 2).

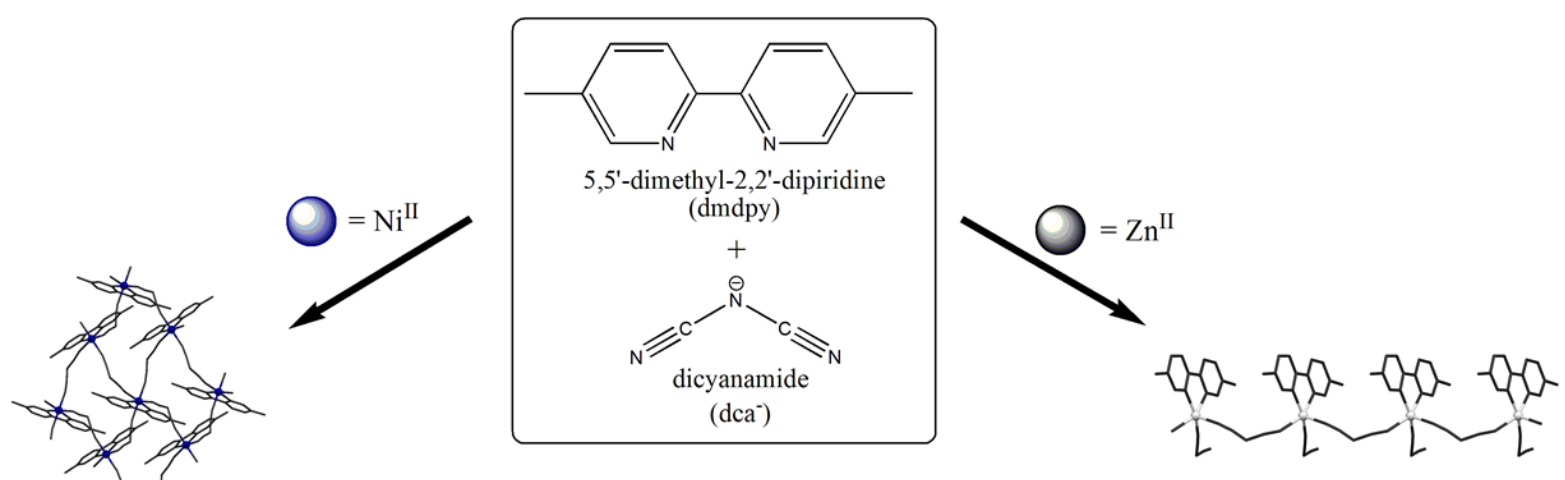

Scheme 2- Formation of the coordination polymers according to the use of the metal ion. 
A fragment of the crystal structure of $\mathbf{1}$ is shown in Figure 1a. Each $\mathrm{Ni}^{\mathrm{il}}$ center is coordinated to six nitrogen atoms from one dmdpy ligand ( $\mathrm{N} 1$ and $\mathrm{N} 1^{i}$ ) and four dca ligands $\mathrm{N} 2$, $\mathrm{N} 2^{\mathrm{i}}, \mathrm{N} 3^{\mathrm{ii}}$ and $\mathrm{N} 3^{\mathrm{iii}}$ in a distorted octahedral geometry. The dmdpy ligand is coordinated to the metal site in a bidentate chelate mode while the dca ligand acts in a bidentate $\mu_{1,5}$ bridge fashion. A tetragonal distortion is evidenced by bond distances values Ni1-N1 (2.092(2) $\AA$ ), Ni1$\mathrm{N} 2(2.075(2) \AA)$ and Ni1-N3 (2.062(3) $\AA$ ) [symmetry codes: $i(1-x, y, 1.5-z) ; i i(1-x, 1-y, 1-z)$ and iii $(x, 1-y, 0.5+z)]$. The bond distances are typical for coordination compounds containing dca and dmdpy ligands.[24] No clear distinction can be made between distance distortions (Table 2). The $\mathrm{N}-\mathrm{Ni}-\mathrm{N}$ angles range from $78.14(9)$ to $94.51(10)^{\circ}$ when the nitrogen atoms are neighbors in the octahedron and are all less than $180^{\circ}$ (when the nitrogen atoms are on opposite summits of the octahedron). Nevertheless, two groups of angles can be distinguished: when two nitrogen atoms are connected by a two-carbon-atom bridge from the dmdpy ligand, the corresponding $\mathrm{N} 1^{i}-\mathrm{Ni}-\mathrm{N} 1$ angle is small $\left(78.14(9)^{\circ}\right)$ and, for independent nitrogen atoms (no direct carbon link), the angles are larger $\left(94.51(10)^{\circ}\right)$. These values demonstrate that the chelating power of the equatorial $\mathrm{N}-\mathrm{C}-\mathrm{C}-\mathrm{N}$ of the dmdpy ligand group leads to smaller angles and to a contraction of the octahedron along the $\mathrm{C}_{2}$ axis of the molecule.

Table 2- Selected bond lengths and angles for (1).

\begin{tabular}{|c|c|c|c|}
\hline \multicolumn{2}{|c|}{ Bond lenghts $(\AA)$} & \multicolumn{2}{|c|}{ Angle $\left(^{\circ}\right)$} \\
\hline Ni1-N1 & $2.092(2)$ & N1-Ni1-N1' & $78.14(9)$ \\
\hline Ni1-N2 & $2.075(2)$ & N1-Ni1-N3" & $94.51(10)$ \\
\hline Ni1-N3" & $2.062(3)$ & N3"-Ni1-N3"' & $92.86(11)$ \\
\hline $\mathrm{Ni1}-\mathrm{N} 1^{i}$ & $2.092(2)$ & $N 1^{i}-\mathrm{Ni1}-\mathrm{N} 3^{i i i}$ & $94.51(10)$ \\
\hline Ni1-N2' & $2.075(2)$ & N2-Ni1-N2' & 179.33(10) \\
\hline Ni1-N3"' & $2.062(3)$ & & \\
\hline $\mathrm{Ni} 1 \cdots \mathrm{Ni} i$ & $8.035(2)$ & & \\
\hline
\end{tabular}

The dca ligands connect the $\mathrm{Ni}^{\prime \prime}$ sites through single bridges along two directions giving

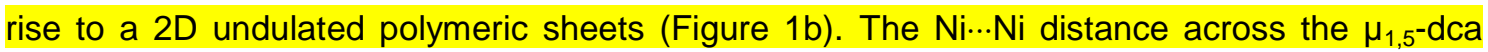
bridges is $8.0353(2) \AA$. By the comparison of this coordination polymer with similar compounds described in the literature, for example, the polymers containing the dca and 2,9-dimethyl-1,10phenantroline ligands published by D. Armentano and coworkers[25] as well as T. Liu and coworkers[26], it is possible to highlight that the ancillary ligand leads to the formation of different networks depending on the position of the methyl substituent position. They observed that the introduction of the methyl substituents ortho to the nitrogen atoms results in the formation of a $(6,3)$ grid. In the present work, if we consider the metal sites as nodes and dca ligands as connectors, the undulated rhombic sheet is topologically described as $(4,4)$ grid. In addition, the values of the $\mathrm{M} \cdots \mathrm{M}$ distances through the single dca bridge is close to the reported values in the cited references. Finally, short $\mathrm{CH} \cdots \pi$ contacts between dmdpy ligand methyl 
group hydrogen atoms and adjacent pyridine rings, with a distance of 2.852 (4) $\AA$, contributed to the stabilization of the crystal packing.

a)

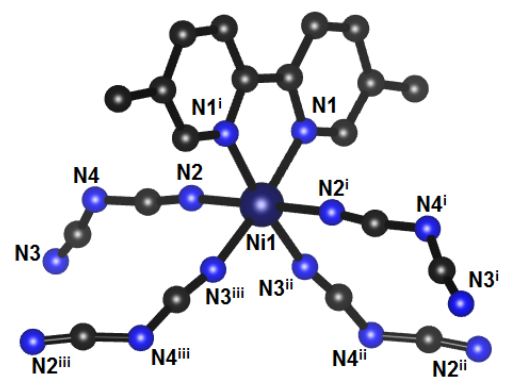

b)

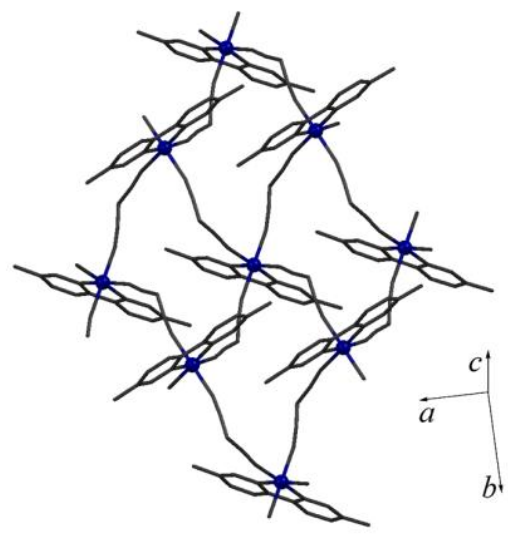

Fig. 1- (a) Fragment of the crystal structure of 1. Symmetry codes: i(1-x,y,1.5-z); ii(1$x, 1-y, 1-z)$ and iii $(x, 1-y, 0.5+z)$. (b) 2D polymer network. Hydrogen atoms are omitted for clarity.

Figure 2a shows a fragment of the crystal structure of $\mathbf{2}$. The zinc ion is coordinated by two nitrogen atoms from one dmdpy ligand and by three nitrogen atoms from three dca ligands. Selected bond lengths and angles are listed in Table 3.

Table 3-Selected bond lengths and angles for 2.

\begin{tabular}{lccc}
\hline \multicolumn{2}{c}{ Bond lenghts $(\AA)$} & \multicolumn{2}{c}{ Angle $\left(^{\circ}\right)$} \\
\hline Zn1-N1 & $2.117(3)$ & N1-Zn1-N2 & $76.55(10)$ \\
Zn1-N2 & $2.140(3)$ & N1-Zn1-N5 & $89.25(12)$ \\
Zn1-N3 & $2.014(3)$ & N2-Zn1-N4 & $91.13(13)$ \\
Zn1-N4 & $2.041(4)$ & N4-Zn1-N5 & $91.13(15)$ \\
Zn1-N5 & $2.086(4)$ & N3-Zn1-N1 & $103.36(13)$ \\
& & N3-Zn1-N2 & $105.62(12)$ \\
& & N3-Zn1-N4 & $101.33(15)$ \\
& & N3-Zn1-N5 & $102.74(13)$ \\
\hline
\end{tabular}

[symmetry codes: $i(-1+x, y, z)]$

The coordination geometry adopted by the $\mathrm{Zn}^{\prime \prime}$ ion is distorted square pyramidal as indicated by the angular structural parameter,[27] $\tau=0.03$. As it was observed for $\mathbf{1}$, the dmdpy ligand is coordinated to the metal site in a bidentate chelate mode. This coordination environment, as well as the values of the bond lengths, can also be found in other structures, including a very similar compound described by D. Mal and coworkers.[28-31] 
The two independent dca ligands exhibit different coordination modes: monodentate and through a bidentate $\mu_{1,5}$ bridge fashion. This ligand connects the $\mathrm{Zn}^{\prime \prime}$ sites along the crystallographic axis $b$ giving rise to a $1 D$ coordination polymer (Figure $2 b$ ). $\pi$ stacking interactions between the dmdpy pyridine rings from interdigitated polymers with a centroid-tocentroid distance of $3.845(14) \AA$ are observed in the crystal packing.

a)

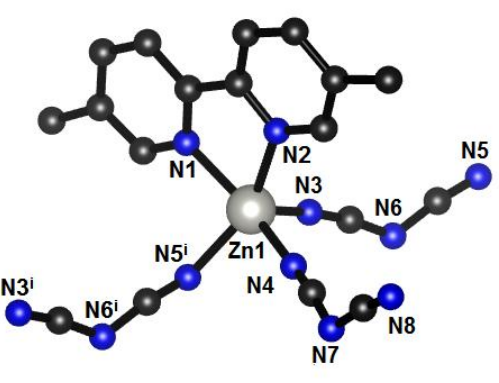

b)

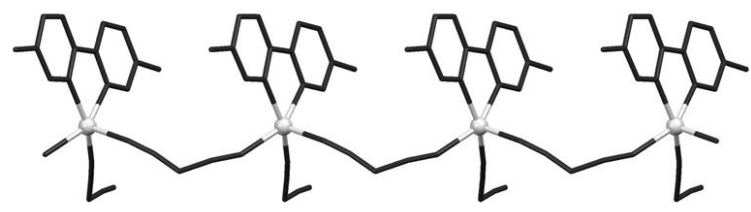

Fig. 2- (a) Fragment of the crystal structure of 2 , symmetry code $i(-1+x, y, z)$. (b) $1 \mathrm{D}$ polymer network. Hydrogen atoms are omitted for clarity.

One of the reasons to obtain two coordination polymers with different dimensionalities is the fact that the $\mathrm{Zn}^{\text {"I }}$ ion has a completely filled $3 d$ orbital. A metal ion with a $d^{10}$ electronic configuration has no ligand field stabilization energy. As a consequence, $\mathrm{Zn}$ " can easily attain variable coordination numbers.[31]

\section{Magnetic properties}

The thermal dependence of the $\chi_{M} T$ product for 1 is shown in Figure 3 . The $\chi_{M} T$ value at $230 \mathrm{~K}$ is $1.05 \mathrm{~cm}^{3} \mathrm{~mol}^{-1} \mathrm{~K}$, close to that calculated for a magnetically isolated $\mathrm{Ni}^{\prime \prime}$ ion with $\mathrm{g}=2.00$ $\left(1.00 \mathrm{~cm}^{3} \mathrm{~mol}^{-1} \mathrm{~K}\right)$. This value remains constant from 230 to $30 \mathrm{~K}$ indicating paramagnetic behavior in this temperature range. Upon cooling, the $\chi_{M} T$ value decreases to a minimum of $0.854 \mathrm{~cm}^{3} \mathrm{~mol}^{-1} \mathrm{~K}$ at $2.5 \mathrm{~K}$ which can be attributed to antiferromagnetic interactions and/or zerofield splitting (ZFS). The $\chi_{M}{ }^{-1}$ versus temperature plot can be fitted to the Curie-Weiss law,

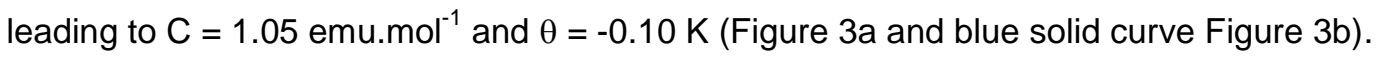



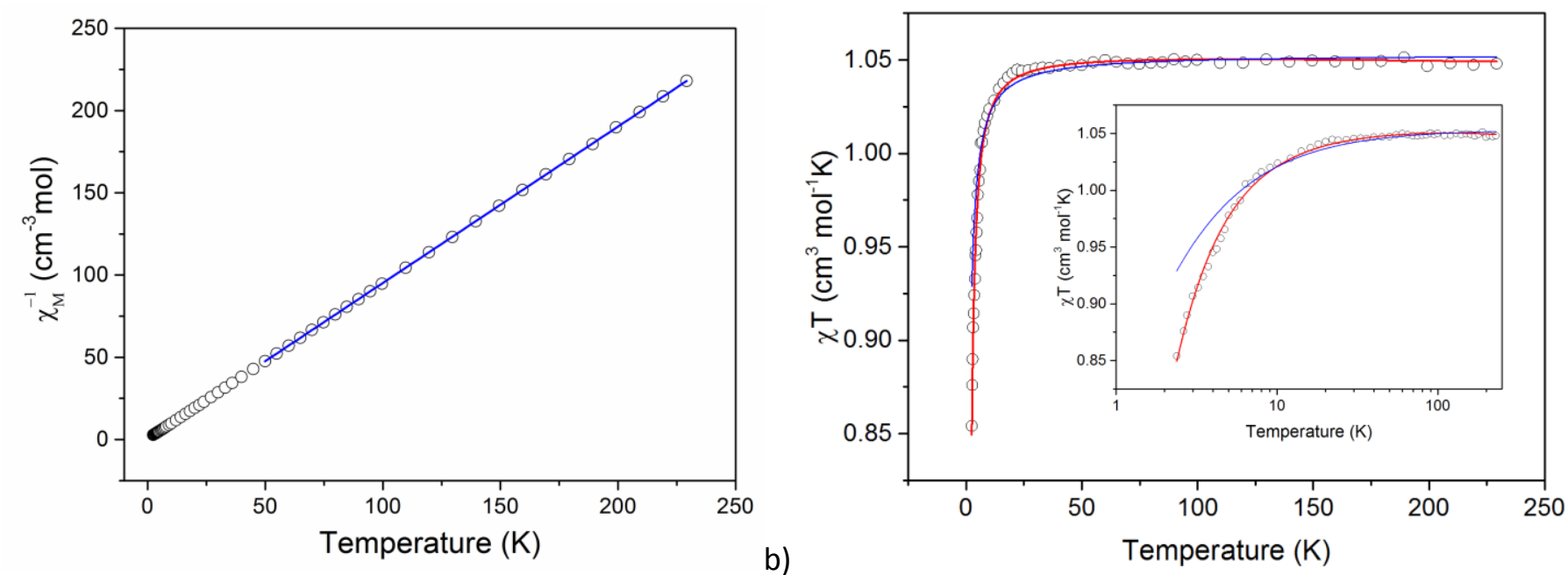

Fig. 3- a) $\chi_{M}{ }^{-1}$ data as a function of temperature fitted to the Curie-Weiss law (solid line). b) Thermal dependence of the $\chi_{M} T$ product for compound 1 . The blue solid curve corresponds to the fit considering only the Curie-Weiss law and the red solid curve is the best fit of experimental data considering the ZFS anisotropy described by eq 1 with the mean field approximation described by eq 2 .

The small Weiss constant value is due to the large distance between $\mathrm{Ni}^{\mathrm{il}}$ ions in the $2 \mathrm{D}$ polymer, and the magnetic properties are best rationalized as arising from an isolated $\mathrm{Ni}^{\mathrm{Il}}$ ion. Despite the fact that the magnetic data can be fitted to the Curie-Weiss law, the quality of the fit improves significantly by the inclusion of ZFS, $D$, as shown in Figure $3 b$ (red solid curve). Therefore, in order to reproduce the experimental data, we employ a model where we treat the on-site anisotropy by the spin Hamiltonian shown in equation (1), then we additionally include the mean field approximation in order to account for the magnetic coupling from nearby $\mathrm{Ni}^{\prime \prime}$ ions,[32] as shown in equation (2).

$$
\hat{H}=g \mu_{B} \vec{B} \cdot \hat{S}+D \hat{S}_{z}^{2}
$$

Here the first term represents the electronic Zeeman interaction and the last term defines the ZFS.

$$
\chi_{m}=\frac{N \beta^{2} g^{2}}{3 k T} S(S+1) \quad \chi_{m}^{M F}=\frac{\chi_{m}}{1-z J^{\prime} \chi_{m}}
$$

Here $z$ is the number of nearest neighbors around a given magnetic molecule in the crystal lattice, and $J$ ' is the interaction parameter between two nearest neighbors. 
The fit of the magnetic data was performed with the MagProp routine, available under DAVE software.[33] The red solid line in Figure $3 b$ shows the best fit curve for compound 1 found with $g=2.05, D=+2.22 \mathrm{~cm}^{-1}$ and the mean field parameter $\lambda=-0.26 \mathrm{emu} / \mathrm{mol}$. The intermolecular magnetic interaction $z J^{\prime}$ is related to $\lambda$ and can be estimated by equation (3).

$$
\lambda=\frac{z J^{\prime}}{g^{2} \mu_{B}^{2}}
$$

The calculated $z J^{\prime}$ value is $-0.23 \mathrm{~cm}^{-1}$, this value is indicative of very weak antiferromagnetic interactions between adjacent $\mathrm{Ni}^{\prime \prime}$ ions. In the present structure, the large $\mathrm{Ni} \ldots \mathrm{Ni}$ separation across the $\mu_{1,5}$-dca bridges (8.035 (2) $\AA$ ) leads to the observed magnetic behavior. This shows the poor ability of the dca bridge to mediate magnetic interactions between the spin carriers when adopting the $\mu_{1,5}$ bridging mode.

As was mentioned in the crystal structure description, there is a compression of the octahedron in 1 that can be the origin of the positive $D$ value observed in the magnetic properties. The data are similar to those observed in other $\mathrm{Ni}^{\mathrm{i}}$-dca coordination polymers.[7, 12]

It is well known that the susceptibility measurements provide only an average value of the g-factors, and the accuracy of this bulk technique in determining spin Hamiltonian parameters in general, and ZFS in particular, is inferior to that of EPR, a spectroscopic technique.[34] Therefore, high-field, high-frequency Electron Paramagnetic Resonance (HFEPR) measurements were performed in order to accurately characterize the g-factors and confirm the ZFS parameters of the $\mathrm{Nil}$ ion in $\mathbf{1}$.

\section{EPR measurements}

In the $\mathrm{O}_{\mathrm{h}}$ point group $\mathrm{Ni}^{l l}$ displays an orbitally non-degenerate ${ }^{3} \mathrm{~A}_{2 \mathrm{~g}}$ ground state with excited orbital-triplet terms, ${ }^{3} \mathrm{~T}_{2 g}(F),{ }^{3} \mathrm{~T}_{1 g}(\mathrm{~F}),{ }^{3} \mathrm{~T}_{1 \mathrm{~g}}(\mathrm{P})$. Spin-orbit coupling permits mixing of the ${ }^{3} \mathrm{~T}_{2 g}$ state into the ground term, but does not lift the spin degeneracy. However, lowering the point-group symmetry from $\mathrm{O}_{\mathrm{h}}$ causes this admixture to be different for each component of the spin-triplet, resulting in ZFS. The magnitude and sign of the axial $2^{\text {nd }}$ order ZFS parameter, $D$, depends on the splitting of the ${ }^{3} \mathrm{~T}_{2 g}$ term, but is inversely proportional to its separation from the ground state. A consequence of these two factors is that the ZFS is highly sensitive to the coordination geometry. The form of the observed spin-triplet $(S=1)$ EPR spectrum depends on the magnitude of the ZFS compared with the applied microwave frequency. [35]

Frequency and temperature dependent studies $(50.8-204.8 \mathrm{GHz}, 5-30 \mathrm{~K})$ were performed. A representative spectrum recorded at $204.8 \mathrm{GHz}$ and $5 \mathrm{~K}$ is displayed in Figure $4 \mathrm{a}$. 


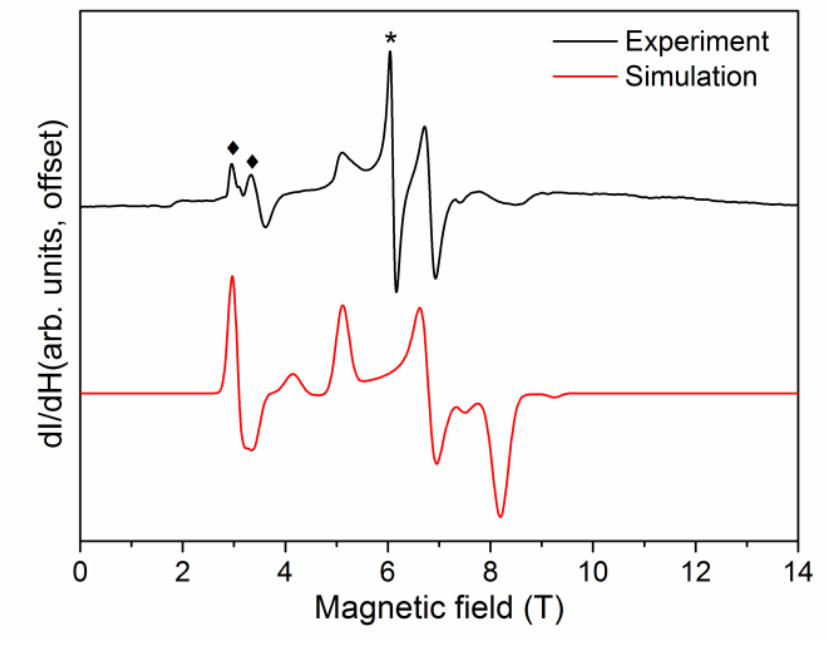

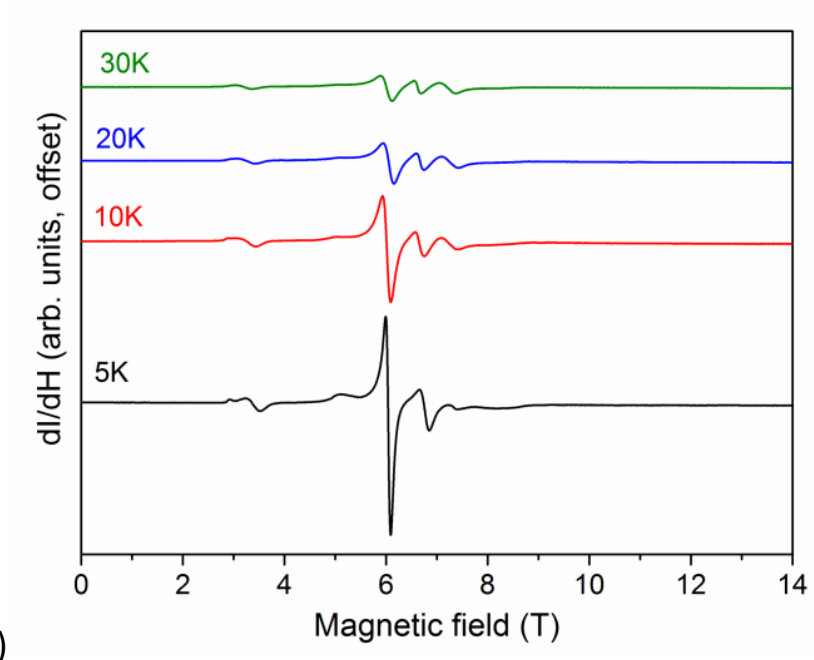

b)

Fig.4-a) High-field powder EPR spectrum (in black) recorded at $204.8 \mathrm{GHz}$ and $\mathrm{T}=5 \mathrm{~K}$, for 1. The simulated spectrum (in red) is displayed below the experimental one. The peaks marked by

( and *) correspond to the double quantum transitions (see main text for explanation). b)

Temperature-dependent powder EPR spectra collected from 5 to $30 \mathrm{~K}$ at $204.8 \mathrm{GHz}$ for 1 .

Most of the resonances observed in the EPR spectrum at $204.8 \mathrm{GHz}$ in Figure 4 can be interpreted as allowed EPR transitions $(\Delta m s= \pm 1)$ within the triplet state, with the exception of the peaks around $3.16 \mathrm{~T}$ (marked by $\bullet$ ) and $6.04 \mathrm{~T}$ (marked by *); these are explained further below.

The spectral simulation was performed using EasySpin[36] and is included below the experimental spectrum in figure 4a). The Hamiltonian described by Eq. (4) was considered:

$$
\hat{H}=g \mu_{B} \vec{B} \cdot \hat{S}+D \hat{S}_{z}^{2}+E\left(\hat{S}_{x}^{2}-\hat{S}_{y}^{2}\right)
$$

The best simulation was achieved using the following parameters: $D=+2.23 \mathrm{~cm}^{-1}, E=0.55$ $\mathrm{cm}^{-1}, g_{x}=2.02, g_{y}=2.02$, and $g_{z}=2.36$. The parameters considered in the simulation are in the range observed for other octahedral $\mathrm{Ni}^{\prime \prime}$ complexes reported in the literature.[9, 35,37-39] The $D$ parameter agrees well with the magnetic measurements.

Based on the temperature dependence (Figure 4b) and Zeeman diagram (Figure S3) it is possible to observe strong resonances at $5.11,6.76$ and $8.19 \mathrm{~T}$, corresponding to excitations from the ground state $\left(m_{s}=-1\right.$ to 0$)$ for $B / / y, x$ and $z$, respectively; here $m_{s}$ represents the projection of the spin, $S$, onto the axis defined by the applied (high) field. Meanwhile, the weaker resonances at 4.15, 7.41 and 9.25 $\mathrm{T}$ are assigned to transitions from the excited state $\left(m_{s}=0\right.$ to $+1)$, for $B / / z, x$ and $y$, respectively. This unambiguously indicates that the $D$ parameter is positive. 
Finally, we explain the two sharp features marked by ( $\bullet$ and *). The resonances (multiple overlapping peaks) clustered around $3.16 \mathrm{~T}$ (marked by ) correspond to normally forbidden double quantum transitions $\left(m_{s}=-1\right.$ to +1$)$ that are allowed in the present case due to the rhombic anisotropy, $E$, that mixes the $m_{s}= \pm 1$ spin sub-levels, giving rise to parallel-mode excitations (we note that the microwaves are not precisely polarized in the employed spectrometer). Meanwhile, the sharp peak observed at $6.04 \mathrm{~T}$ corresponds to a two-photon transition involving all-three triplet levels. Such two-photon transitions are often observed in octahedral $\mathrm{Ni}^{\mathrm{il}}$ compounds $[9,35,37]$ and are not accounted for in the EasySpin simulations. They occur for certain orientations of the applied field at which the triplet states are equidistant in energy (harmonic). Based on the best simulation parameters, the position of this peak is well reproduced.

\section{Conclusion}

We reported the synthesis, characterization and crystal structure of two new coordination polymers containing the 5,5'-dimethyl-2,2'-dipyridine ligand, dicyanamide anion and transition metals $\mathrm{Ni}^{\prime \prime}$ and $\mathrm{Zn}$ ". Compounds $\mathbf{1}$ and $\mathbf{2}$ are $2 \mathrm{D}$ and $1 \mathrm{D}$ coordination polymers, respectively. The magnetic properties of $\mathbf{1}$ were investigated by susceptibility measurements and very weak antiferromagnetic interactions were observed between the $\mathrm{Ni}^{\mathrm{il}}$ ions. The ZFS parameters of the $\mathrm{Ni}^{\prime \prime}$ ion in $\mathbf{1}$, as well as its $\mathrm{g}$-tensor, were characterized by HFEPR measurements.

\section{Acknowledgements}

The authors thank the Brazilian agencies CNPq, CAPES, FAPERJ and FAPEMIG for financial support and LabCri (Universidade Federal de Minas Gerais) for the use of crystallographic facilities. We also thank Professor Miguel A. Novak for magnetic measurements. Work performed at the NHMFL is supported by the NSF (DMR-1157490 and DMR-1309463) and the State of Florida. 


\section{References}

[1] T. Yu, S. Wang, X. Li, X. Gao, C. Zhou, J. Cheng, B. Li, J. Li, J. Chang, H. Hou, Z. Liu, CrystEngComm, 18 (2016) 1350-1362.

[2] L.L. Zheng, C.X. Zhou, S. Hu, A.J. Zhou, Polyhedron, 104 (2016) 91-98.

[3] Y.-D. Zhang, S.-H. Zhang, C.-M. Ge, Y.-G. Wang, Y.-H. Huang, H.P. Li, Inorganic, MetalOrganic, and Nano-Metal Chemistry, 43 (2013) 990-994.

[4] J.-M. Hu, K. Van Hecke, B. Yu, G.-H. Cui, Inorganic Chemistry Communications, 61 (2015) 217-220.

[5] C. Hopa, I. Cokay, Acta Crystallographica Section C Structural Chemistry, 72 (2016) 149154.

[6] J.H. Martin Krizan, Jaromır Vinklarek, Zdenka Ruzickova, Milan Erben, New J. Chem., 39 (2015) 576--588

[7] L.K. Das, C.J. Gómez-García, A. Ghosh, Dalton Trans., 44 (2015) 1292-1302.

[8] J.-T. Lu, D.-D. Meng, Q.-G. Meng, Acta Crystallographica Section C Structural Chemistry, 72 (2016) 99-104.

[9] O.V. Nesterova, S.R. Petrusenko, V.N. Kokozay, B.W. Skelton, J. Jezierska, W. Linert, A. Ozarowski, Dalton Transactions, (2008) 1431-1436.

[10] X.-Y. Liu, L.-Q. Duan, Q. Wei, S.-P. Chen, Inorganica Chimica Acta, 423 (2014) 462-468.

[11] H. Ogawa, K. Mori, K. Murashima, S. Karasawa, N. Koga, Inorganic Chemistry, 55 (2016) 717-728.

[12] S. Kundu, S. Roy, K. Bhar, R. Ghosh, C.-H. Lin, J. Ribas, B.K. Ghosh, Journal of Molecular Structure, 1038 (2013) 78-85.

[13] L.B. Lopes, C.C. Corrêa, G.P. Guedes, M.G.F. Vaz, R. Diniz, F.C. Machado, Polyhedron, 50 (2013) 16-21.

[14] Oxford, CrysAlisRED, Oxford Diffraction Ltd Abingdon, U.K., 2008.

[15] G.M. Sheldrick, Acta Crystallogr., A64 (2008) 112-122.

[16] A.C. Larson, Crystallographic Computing, in: S.R.H.C.P.H. F. R. Ahmed (Ed.), Copenhagen, Munksgaard., 1970.

[17] R.H. Blessing, Acta Crystallogr., Sect. A 51 (1995) 33.

[18] K.M.a.F. Izumi, VESTA 3 for three-dimensional visualization of crystal, volumetric and morphology data, Journal of Applied Crystallography, 44, 1272-1276, 2011.

[19] P.R.E. C.F. Macrae, P. McCabe, E. Pidcock, G.P. Shields, R. Taylor, M. Towler, J. van De Streek, J. Appl. Crystallogr. , 39 (2006) 453-457.

[20] A.K. Hassan, L.A. Pardi, J. Krzystek, A. Sienkiewicz, P. Goy, M. Rohrer, L.C. Brunel, Journal of Magnetic Resonance, 142 (2000) 300-312.

[21] K. Bhar, S. Das, S. Satapathi, P. Mitra, J. Ribas, B.K. Ghosh, Polyhedron, 29 (2010) 20412047.

[22] K. Bhar, S. Choubey, P. Mitra, G. Rosair, J. Ribas, B.K. Ghosh, Journal of Molecular Structure, 988 (2011) 128-135.

[23] A. Das, C. Marschner, J. Cano, J. Baumgartner, J. Ribas, M.S. El Fallah, S. Mitra, Polyhedron, 28 (2009) 2436-2442.

[24] T. Ghosh, J. Adhikary, P. Chakraborty, P.K. Sukul, M.S. Jana, T.K. Mondal, E. Zangrando, D. Das, Dalton Trans., 43 (2014) 841-852.

[25] D. Armentano, G. De Munno, F. Guerra, M. Julve, F. Lloret, Inorganic Chemistry, 45 (2006) 4626-4636

[26] T.Liu, Z. Bao, L.Li, Journal of Coordination Chemistry, 64 (2011) 1063-1068.

[27] L. Yang, D.R. Powell, R.P. Houser, Dalton Trans., (2007) 955-964.

[28] X.-L. Yu, Y. Fang, C.-Y. Huang, Y.-P. Gu, W.-S. You, Acta Crystallographica Section E Structure Reports Online, 63 (2007) m358-m359.

[29] P. Chakraborty, J. Adhikary, S. Samanta, D. Escudero, A.C. Castro, M. Swart, S. Ghosh, A. Bauzá, A. Frontera, E. Zangrando, D. Das, Crystal Growth \& Design, 14 (2014) 4111-4123.

[30] M. Maiti, D. Sadhukhan, S. Thakurta, S. Roy, G. Pilet, R.J. Butcher, A. Nonat, L.J. Charbonnière, S. Mitra, Inorganic Chemistry, 51 (2012) 12176-12187.

[31] D. Mal, R. Sen, P. Brandão, Z. Lin, Polyhedron, 53 (2013) 249-257.

[32] P. Stachová, M. Korabik, M. Koman, M. Melník, J. Mrozinski, T. Glowiak, M. Mazúr, D. Valigura, Inorganica Chimica Acta, 359 (2006) 1275-1281.

[33] R.T. Azuah, L.R. Kneller, Y. Qiu, P.L.W. Tregenna-Piggott, C.M. Brown, J.R.D. Copley, and R.M. Dimeo, J. Res. Natl. Inst. Stan. Technol. 114, (2009) 341-358. 
[34] J. Krzystek, A. Ozarowski, J. Telser, Coordination Chemistry Reviews, 250 (2006) 23082324.

[35] M.H. David Collison, Victoria M. Jones, Frank E. Mabbs, Eric J. L. McInnes, Peter C. Riedi, Graham M. Smith, Robin G. Pritchardd and Wendy I. Cross, J. Chem. Soc., Faraday T rans., 94 (1998) 3019-3025.

[36] S. Stoll, A. Schweiger, EasySpin, a comprehensive software package for spectral simulation and analysis in EPR, Journal of Magnetic Resonance, 178 (2006) 42-55.

[37] A.A.K.K. Pieter J. van Dam, Eduard J. Reijerse, and Wilfred R. Hagen, Journal of Magnetic Resonance, 130 (1998) 140-144.

[38] R.B. Radovan Herchel, J. Krzystek, Andrew Ozarowski, Marc Duran, and Joris van Slageren,J. Am. Chem. Soc. , 129 (2007) 10306-10307.

[39] A.S. J. Mrozinskia, A. Pochaba, Y. Dromzee,M. Verdaguer, E. Goovaerts, H. Varcammen, B. Korybut-Daszkiewicz, Journal of Molecular Structure 559 (2001) 107-118. 


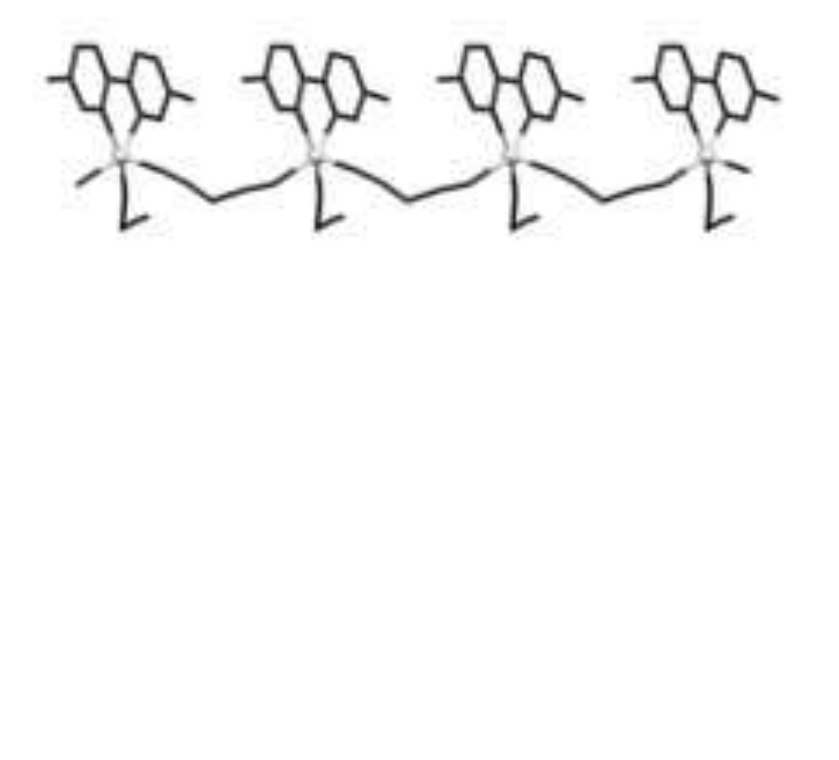

$20-2 a-2 a-2 a$

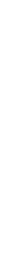

\section{Graphical Abstract - pictogram}

-

年

(


Graphical Abstract- Synopsis

Two new compounds $\left[\mathrm{Ni}(\mathrm{dca})_{2}(\mathrm{dmdpy})\right]$ (1) and $\left[\mathrm{Zn}(\mathrm{dca})_{2}(\mathrm{dmdpy})\right]$ (2), (dca = dicyanamide and dmdpy = 5,5'-dimethyl-2,2'-dipyridine) were synthesized and characterized by single crystal X-ray diffraction, FTIR and thermal analysis. In both compounds, the dmdpy ligand adopts a bidentate chelating coordination mode while dca anions act in a $\mu_{1,5}$ bridging fashion. The magnetic properties of $\mathbf{1}$ were studied by magnetic susceptibility and high field/frequency electron paramagnetic resonance (HFEPR) measurements. 